\title{
Analytical solution of energy eigen value, eigen function and angular wave function of Dirac equation with Rosen Morse plus Rosen Morse potential in terms of Romanovski polynomials for exact spin symmetry
}

\author{
Ihtiari Prastyaningrum ${ }^{1}$, C. Cari $^{2}$, A. Suparmi ${ }^{3}$ \\ ${ }^{1}$ Teaching and Education Institute PGRI Madiun \\ ${ }^{2,3}$ Physics Department Sebelas Maret University \\ Email: ihtiari.prastya8911@gmail.com
}

Received 7 October 2016, Revised 28 November 2016, Accepted 30 December 2016

\begin{abstract}
The energy eigenvalues and eigenfunctions of Dirac equation for Rosen Morse plus Rosen Morse potential are investigated numerically in terms of finite Romanovsky Polynomial. The bound state energy eigenvalues are given in a closed form and corresponding eigenfunctions are obtained in terms of Romanovski polynomials. The energi eigen value is solved by numerical method with Matlab 2011.
\end{abstract}

Keyword : Dirac equation; Rosen Morse potential with the centrifugal term; Romanovsky polynomials.

\section{Introduction}

It is well known that the exact energy eigenvalues of the bound state play an important role in quantum mechanics. In particular, the Dirac equation which describes the motion of a spin-1/2 particle has been used in solvingmany problems of nuclear and high-energy physics (Azizi \& Rajabi, 2013). The exact solution of some non-central potentials for $l$-wave have been investigated intensively by some authors (Cari \& Suparmi, 2012). For example on 2004, Reity, Rubish and Myhalyna was investigated Dirac equation for vector and scalar potential (Reity \& Myhalyna, 2014), Victor M. Villalba find the solution in the presence of a gravitational instanton on 2005 (Villaba, 2005), and Bakkeshizadeh and Vahidi solving the Dirac equation for Coulomb and NAD potential (Bakkeshizadeh \& Wahidi, 2012).

In general the non-central potential is a potential as a function of radial and angular positions simultaneously. These potentials is constructed by combination of radial shape or nonshape invariance. The potentials that can be used sucs as hyperbolic Scarf Potential, Manning Rosen, Rosen Morse, etc. The bound state energy spectra of these potentials have been investigated by Supersymetri method (Cari, Suparmi \& H. Marini, 2012), Nikivorof-Uvarof (Ikot \& Akpabio, 2010), Laplace Transformation Method (Eshghi, Hamzavi \& Ikhdair, 2012). Romanovsky polynomials (David, 2009), WKB Method (Sadeghi, Pahlavani, Naderi, et al., 2005). 
In this paper will be solved the the Dirac equation for non-central potential in term of Romanovski polynomials by numerical method. The Dirac equation of non-central potentials are solved using separation variable method when the non-central potential is separable. We must set the Dirac equation like hypergeometric term and then make a differential equation in term of Romanovsky polynomials. The last we will get a complicated equation of energi eigen value that can't be solved by analitical method so we must use numerical method to find of solution. Beside that we also get angular wave function and that must be solved by Matlab 2011.

\section{Basic Theory}

\subsection{Dirac Equation}

The relativistic Dirac equation is a covariant first order differential equation in a four dimensional space-time representation. In The one dimensional Dirac equation, Solution can be simplified by adopting of two components approach. These components contain solution for positive and negative energy spinors (Weber, 2007). Dirac equation with the scalar potential $S(r)$ dan vector potential $V(r)$ is given as,

$\left\{c \boldsymbol{\alpha} \cdot \boldsymbol{p}+\beta\left(m c^{2}+S(\boldsymbol{r})\right)\right\} \psi(\boldsymbol{r})=\{E-V(\boldsymbol{r})\} \psi(\boldsymbol{r})$

Where $\mathrm{M}$ is rest mass of particle, $\mathrm{E}$ is the total energy, and $\mathbf{p}$ is the momentum operator. By setting $\psi(\boldsymbol{r})=\left(\begin{array}{l}\zeta(r) \\ \chi(r)\end{array}\right)$ and using Puli's matrices, we can get the form of equation (1) equal,

$c \sigma \cdot \boldsymbol{p} \zeta(r)=\left\{M c^{2}+S(\boldsymbol{r})+E-V(\boldsymbol{r})\right\} \chi(r)$

When scalar potential is equal to vector potential, $S(r)=V(r)$ we get corelation,

$c \sigma \cdot \boldsymbol{p} \frac{c \sigma \cdot \boldsymbol{p} \zeta(r)}{M c^{2}+E}=\left\{-M c^{2}+E-2 V(\boldsymbol{r})\right\} \zeta(r)$

By applying Pauli matrices it is simply shown that $(\sigma \cdot p)(\sigma \cdot p)=p^{2}$ and if $\hbar, c=1$ so equation (3) can be rewriten as,

$\left\{p^{2}-2 V(M+E)(\boldsymbol{r})\right\} \zeta(r)=\left\{E^{2}-M^{2}\right\} \zeta(r)$

Equation (3) is Dirac equation where scalar potential is similar to the vector potential, $\mathrm{M}$ is the relativistic mass and $\mathrm{E}$ is relativistic energy. In non relativistic limit, $E-M \rightarrow E_{N R}, E_{N R}$ is non relativistic energy, $E+M \rightarrow 2 \mu$, where $\mu$ is the non relativistic mass. By applying that condition into equation (4) so that equation can be reduced to,

$\left\{p^{2}-2 V 2 \mu(\boldsymbol{r})\right\} \zeta(r)=\{E-M\} 2 \mu \zeta(r)$

$\left\{\frac{p^{2}}{2 \mu}-2 V(\boldsymbol{r})\right\} \zeta(r)=E_{N R} \zeta(r)$

\subsection{Rosen Morse Potential}

Rosen Morse potential given as,

$V(r)=\left(\frac{v(v+1)}{\sin ^{2} r}-2 q \cot r\right)$

Where $v$ and $q$ is constant. If $r \rightarrow i r, q \rightarrow i q$ so equation (6) can be rewriten as,

$V(r)=\left(-\frac{v(v+1)}{\sinh ^{2} r}-2 q \operatorname{coth} r\right)$ 
Equation (7) is Eckart potential. Is showed that Rosen Morse potential is compleks form of Eckart potential.

\section{Analysis Method}

The method that be used is Romanovski polynomials. Romanovski polynomials were discovered in 1884 by Routh in the form of complexified Jacobi polynomials on the unit circle in the complex plane and were then rediscovered as real polynomials by Romanovski in a statistics framework (Ikot \& Akpabio (2010). The Romanovski polynomials are built from The generalized hypergeometric equation is given,

$\sigma(x) \frac{d^{2} y_{n}(x)}{d x^{2}}+\tau(x) \frac{d y_{n}(x)}{d x}-\lambda y_{n}(x)=0$

where

$\sigma(x)=a x^{2}+b x+c ; \quad \tau=d x+e$ and $\lambda_{n}=-(n(n-1)+2 n(1-p))$

For Romanovski polynomial, the values of parameters in equation (8) are, $a=1, b=0, c=1, d=2(1-p) ; e=q, p>0 ; y_{n}(x)=D_{n}^{(p, q)}(x) ; p=-\beta>n$

So we get the new type of differential equation is caled Romanovski polynomials.

$\left(1+x^{2}\right) \frac{d^{2} D_{n}^{(p, q)}(x)}{d x^{2}}+(2 x(1-p)+q) \frac{d D_{n}^{(p, q)}(x)}{d x}-\{n(n-1)+2 n(1-p)\} D_{n}^{(p, q)}(x)=$ 0

The weight function obtained by solving the Pearson differential equation is

$\frac{d}{d x}(\sigma(x) w(x))=\tau(x) w(x)$

And

$w(x)=\exp \left(\int \frac{(d-2 a) x+(e-b)}{a x^{2}+b x+c} d x\right)$

The corresponding polynomials to the weight function equation (12) are built up from the Rodrigues representation that is given as,

$D_{n}^{(p, q)}(x)=\frac{1}{w(x)} \frac{d^{n}}{d x^{n}}\left(\left(1+x^{2}\right)^{n} w(x)\right)$

By inserting all the parameter into equation (12) we obtain the weight function as,

$w(x)=\left(1+x^{2}\right)^{-p} e^{q \tan ^{-1}(x)}$

The Romanovski polynomials obtain by inserting equation (14) in to equation (13) as,

$D_{n}^{(p, q)}(x)=\frac{1}{\left(1+x^{2}\right)^{-p} e^{q \tan ^{-1}(x)}} \frac{d^{n}}{d x^{n}}\left(\left(1+x^{2}\right)^{n}\left(1+x^{2}\right)^{-p} e^{q \tan ^{-1}(x)}\right)$

\section{Solution of Radial Dirac Equation for Non-central Potential using Romanovsky Polynomials}

One dimension of Dirac equation with Rosen Morse potential plus Rosen Morse potential with the centrifugal term is showed in equation (16),

$\frac{\partial}{\partial r}\left(r^{2} \frac{\partial R(r)}{\partial r}\right)-r^{2}\left\{(E+M) \gamma^{2}\left(\frac{v(v+1)}{\sin ^{2} \gamma r}-2 q \tan \gamma r\right)-\left(E^{2}-M^{2}\right)\right\} R(r)=\lambda R(r)$

Where $\lambda=l(l+1)$ if we set that,

$\frac{1}{r^{2}} \cong \gamma^{2}\left(d_{0}+\frac{1}{\sin ^{2} \gamma r}\right)$

with $d_{0}=\frac{1}{12}$, if we set $\mathrm{R}(r)=\frac{\chi(r)}{r}$, equation (16) can be rewriten as, 
$\frac{\partial^{2} \chi(r)}{\partial r^{2}}-$

$\gamma^{2}\left\{\left(\frac{v(v+1)(E+M)+l(l+1)}{\sin ^{2} \gamma r}-2(E+M) q \cot \gamma r\right)-\frac{\left(E^{2}-M^{2}\right)}{\gamma^{2}}+\left(l(l+1) d_{0}\right)\right\} \chi(r)=0$

By making an approriate change of variable, $\cot \gamma r=x$, then equation (18) becomes

$\left(1+x^{2}\right) \frac{d^{2}}{d x^{2}}+2 x \frac{d}{d x}-\left\{\frac{l(l+1) d_{0} \gamma}{\left(1+x^{2}\right)}-\frac{2(E+M) q \gamma x}{\left(1+x^{2}\right)}-\frac{\left(E^{2}-M^{2}\right)}{\gamma\left(1+x^{2}\right)}+(E+M) v(v+1) \gamma+\right.$

$l(l+1) \gamma\} \chi(r)=0$

To solve equation (19) in term Romanovski polynomials, equation (14) suggest the substitution in equation (19) as,

$\chi_{n}=\left(1+x^{2}\right)^{\frac{p}{2}} e^{-\frac{\alpha}{2} \tan ^{-1} x} D_{n}^{(\beta, \alpha)}(x)$

By inserting equation (20) into (19) we obtain,

$$
\begin{aligned}
& \left(x^{2}+1\right) \frac{\partial^{2} D_{n}^{(p, q)}(x)}{\partial x^{2}}+(2 x(\beta+1)-\alpha) \frac{\partial D_{n}^{(p, q)}(x)}{\partial x}+ \\
& \left\{\left(\frac{-\beta^{2}-\alpha \beta x+\frac{\alpha^{2}}{4}+(E+M) 2 q \gamma x+\frac{\left(E^{2}-M^{2}\right)}{\gamma}-l(l+1) d_{0} \gamma}{\left(1+x^{2}\right)}\right)+\beta+\beta^{2}-(E+M) v(v+1) \gamma-\right. \\
& l \gamma(l+1)\} D_{n}^{(p, q)}(x)=0
\end{aligned}
$$

By setting the coefficient of $\frac{1}{1+x^{2}}$ in equation (21) to be zero, that are

$-\alpha \beta+(E+M) 2 q \gamma=0$

$-\beta^{2}+\frac{\alpha^{2}}{4}+\frac{\left(E^{2}-M^{2}\right)}{\gamma}-l(l+1) d_{0} \gamma=0$

Then equation (21) reduces to the Romanovski equation like in equation (10) and then comparing the parameters between equations (10) and (21) we obtain the relation,

$(\beta+1)=(-p+1)$ dan $\alpha=-q$

$l \gamma(l+1)+(E+M) v(v+1) \gamma-\left[\beta+\frac{1}{2}\right]^{2}+\frac{1}{4}=n(n-1)+2 n(1-p)$

From equation (23b) we have

$\beta=\sqrt{l \gamma(l+1)+(E+M) v(v+1) \gamma+\frac{1}{4}}-n-\frac{1}{2}$

And from equation (22b) we obtain

$\alpha^{2}=-2\left(\frac{\left(E^{2}-M^{2}\right)}{\gamma}-l(l+1) d_{0} \gamma\right) \pm 2 \sqrt{\left(\frac{\left(E^{2}-M^{2}\right)}{\gamma}-l(l+1) d_{0} \gamma\right)^{2}+(E+M)^{2} 4 q^{2} \gamma^{2}}$

$\beta^{2}=\frac{\alpha^{2}}{4}+\frac{\left(E^{2}-M^{2}\right)}{\gamma}-l(l+1) d_{0} \gamma$

Using equation (24) and (25b) we can obtain

$\alpha=-\frac{(E+M) 2 q \gamma}{\sqrt{l \gamma(l+1)+(E+M) v(v+1) \gamma+\frac{1}{4}-n-\frac{1}{2}}}$

And finally we obtain the energy spectra as, 


$$
\begin{aligned}
& \left(E^{2}-M^{2}\right)=\gamma\left(\sqrt{l \gamma(l+1)+(E+M) v(v+1) \gamma+\frac{1}{4}}-n-\frac{1}{2}\right)^{2}- \\
& \frac{\gamma}{4}\left(-\frac{(E+M) 2 q \gamma}{\sqrt{l \gamma(l+1)+(E+M) v(v+1) \gamma+\frac{1}{4}-n-\frac{1}{2}}}\right)^{2}+l(l+1) d_{0} \gamma^{2}
\end{aligned}
$$

To find the energi eigen value from equation (27) we can't use analitical method, but we must solve the equation by numerical method. By numerical method with variation of gamma and $\mathrm{n}$ value we get the energi eigen value that be showed at the table bellow,

Table 1. Energi eigen value with $n$ and $\gamma$ variation

\begin{tabular}{c|c|c|c|c|c}
\hline \multicolumn{6}{c}{$\mathrm{v}=1, \mu=1, M=1, m=2$ and $\gamma=1,0037$} \\
\hline$n$ & 1 & 2 & 3 & 4 & 5 \\
\hline$E\left(\mathrm{fm}^{-1}\right)$ & 5.3924 & 6.4945 & 7.5211 & 8.6292 & 9.6785 \\
\hline $\mathrm{v}=1, \mu=1, M=1, m=2$ and $\gamma=1,0503$ \\
\hline$n$ & 1 & 2 & 3 & 4 & 5 \\
\hline$E\left(\mathrm{fm}^{-1}\right)$ & 4.5153 & 5.5153 & 6.4874 & 7.4422 & 8.3858 \\
\hline $\mathrm{v}=1, \mu=1, M=1, m=2$ and $\gamma=1,1045$ \\
\hline$n$ & 1 & 2 & 3 & 4 & 5 \\
\hline$E\left(\mathrm{fm}^{-1}\right)$ & 3.8309 & 4.7476 & 5.6355 & 6.5064 & 7.3655 \\
\hline
\end{tabular}

And with variation of $n, v$, and $q$ we can see on the Table 2 .

Table 2. Energy eigen value with $v$ and $n$ variation

\begin{tabular}{lccccc}
\hline $\mathbf{n}$ & $\boldsymbol{v}$ & $\boldsymbol{q}$ & $\mathbf{M}$ & $\boldsymbol{\gamma}$ & $\mathbf{E}\left(\boldsymbol{f m}^{-\boldsymbol{I}}\right)$ \\
\hline $\mathbf{1}$ & 0.1 & 0 & 2 & 1 & 4.3966 \\
$\mathbf{1}$ & 0.2 & 0 & 2 & 1 & 4.0012 \\
$\mathbf{1}$ & 0.3 & 0 & 2 & 1 & 3.5876 \\
$\mathbf{1}$ & 0.4 & 0 & 2 & 1 & 3.2185 \\
$\mathbf{2}$ & 0.1 & 0 & 2 & 1 & 6.0956 \\
$\mathbf{2}$ & 0.2 & 0 & 2 & 1 & 5.5301 \\
$\mathbf{2}$ & 0.3 & 0 & 2 & 1 & 4.9456 \\
$\mathbf{2}$ & 0.4 & 0 & 2 & 1 & 4.3809 \\
$\mathbf{3}$ & 0.1 & 0 & 2 & 1 & 7.8766 \\
$\mathbf{3}$ & 0.2 & 0 & 2 & 1 & 7.1464 \\
$\mathbf{3}$ & 0.3 & 0 & 2 & 1 & 6.3936 \\
$\mathbf{3}$ & 0.4 & 0 & 2 & 1 & 5.0034 \\
$\mathbf{4}$ & 0.1 & 0 & 2 & 1 & 9.6894 \\
$\mathbf{4}$ & 0.2 & 0 & 2 & 1 & 8.7949 \\
$\mathbf{4}$ & 0.3 & 0 & 2 & 1 & 7.8762 \\
$\mathbf{4}$ & 0.4 & 0 & 2 & 1 & 6.9902 \\
\hline
\end{tabular}

Beside using variation of $\gamma, v$ and $n$ value we can calculate the enrgy eigen value with $q$ variation. The result is showed by Table 3 . 
Table 3. Energy eigen value with $q$ and $n$ variation

\begin{tabular}{cccccc}
\hline $\mathbf{n}$ & $\boldsymbol{v}$ & $\boldsymbol{q}$ & $\mathbf{M}$ & $\boldsymbol{\gamma}$ & $\mathbf{E}(\mathbf{e V})$ \\
\hline $\mathbf{1}$ & 0 & 0.01 & 2 & 1 & 4.5979 \\
$\mathbf{1}$ & 0 & 0.02 & 2 & 1 & 4.1954 \\
$\mathbf{1}$ & 0 & 0.03 & 2 & 1 & 3.5859 \\
$\mathbf{1}$ & 0 & 0.04 & 2 & 1 & 2.8955 \\
$\mathbf{2}$ & 0 & 0.01 & 2 & 1 & 6.4173 \\
$\mathbf{2}$ & 0 & 0.02 & 2 & 1 & 5.9308 \\
$\mathbf{2}$ & 0 & 0.03 & 2 & 1 & 5.1475 \\
$\mathbf{2}$ & 0 & 0.04 & 2 & 1 & 4.2377 \\
$\mathbf{3}$ & 0 & 0.01 & 2 & 1 & 8.3101 \\
$\mathbf{3}$ & 0 & 0.02 & 2 & 1 & 7.7075 \\
$\mathbf{3}$ & 0 & 0.03 & 2 & 1 & 6.6709 \\
$\mathbf{3}$ & 0 & 0.04 & 2 & 1 & 5.4624 \\
$\mathbf{4}$ & 0 & 0.01 & 2 & 1 & 10.2320 \\
$\mathbf{4}$ & 0 & 0.02 & 2 & 1 & 9.4778 \\
$\mathbf{4}$ & 0 & 0.03 & 2 & 1 & 8.0960 \\
$\mathbf{4}$ & 0 & 0.04 & 2 & 1 & 6.5254 \\
\hline
\end{tabular}

From Table 1 we can invetigated the graph relation of energy and $n$ like figure 1 .

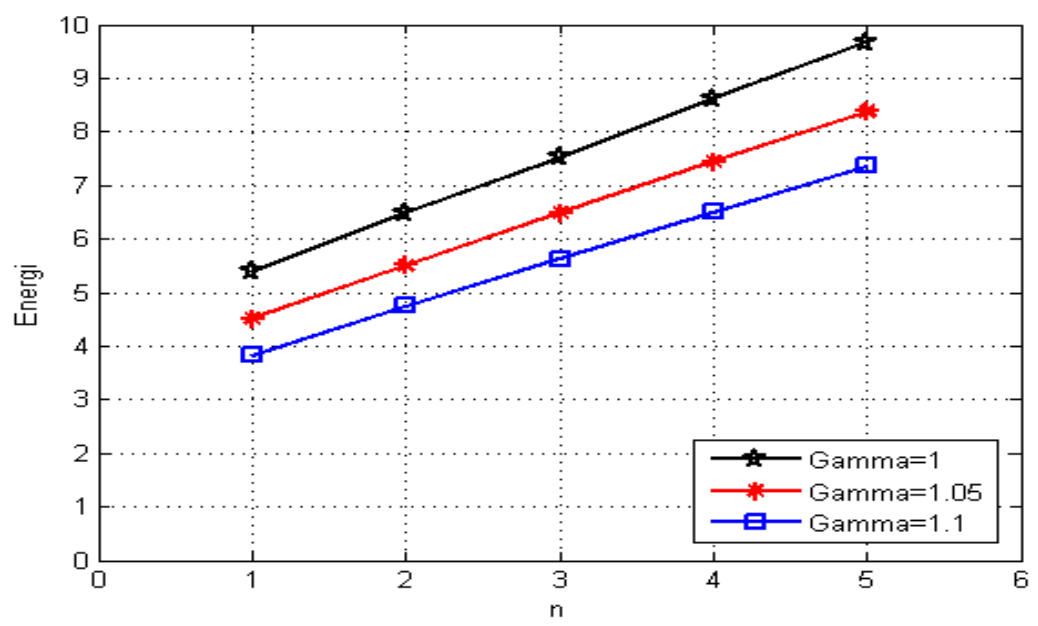

Figure 1. Graph of energy eigen value versus $n$

From the graph can be showed that if the $n$ value is increase so the energi eigen value also increase. The fact olso can be applied to $\gamma$ value. With little increasing of $\gamma$ can make large change of energy eigen value. The increasing of $n$ show that if elektron stay far from the nucleus, it need the bigger energy. But if the electron distrubing by $v$ and $q$ the energy is decrease.

To determine the radial wave function, equations (23a), (24) and (26) are inserted in to equations (14) and (15) so that we obtain the weight function $w(x)$ and the Romanovski polynomials $D_{n}^{(p, q)}(x)$ as

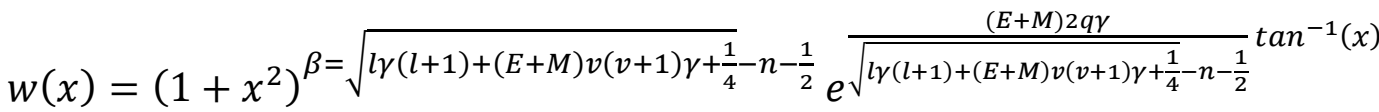

And

$D_{n}^{(p, q)}(x)=\mathcal{R}_{n}^{(-\beta,-\alpha)}(x)=\frac{1}{\left(1+x^{2}\right)^{\beta} e^{-\alpha \tan ^{-1}(x)}} \frac{d^{n}}{d x^{n}}\left(\left(1+x^{2}\right)^{\beta} e^{-\alpha \tan ^{-1}(x)}\right)$ 
And the result of the wave function of the nth level is

$X_{n ; l}=\sqrt{\left(1+x^{2}\right)^{\beta} e^{-\alpha \tan ^{-1}(x)}} \mathcal{R}_{n}^{(-\beta,-\alpha)}(x)$

Some the solving of equation (29) and (30) are

$\mathcal{R}_{0}{ }^{\left(-\beta_{0},-\alpha_{0}\right)}=1$

$X_{0 ; l}=\sqrt{\left(1+x^{2}\right)^{\beta} e^{-\alpha \tan ^{-1}(x)}}$

$\mathcal{R}_{1}{ }^{\left(-\beta_{1},-\alpha_{1}\right)}=2 x(\beta+1)-\alpha$
$X_{1 ; l}=\sqrt{\left(1+x^{2}\right)^{\beta} e^{-\alpha \tan ^{-1}(x)}}(2 x(\beta+1)-\alpha)$

$\mathcal{R}_{2}{ }^{\left(-\beta_{2},-\alpha_{2}\right)}=4(\beta+2)^{2} x^{2}+2(\beta+2)\left(1+x^{2}\right)-4(\beta+2) x^{2}-4(\beta+2) \alpha x+2 \alpha+$ $\alpha^{2}$

$$
\begin{gathered}
X_{3 ; l}=\sqrt{\left(1+x^{2}\right)^{\beta} e^{-\alpha \tan ^{-1}(x)}}\left(4(\beta+2)^{2} x^{2}+2(\beta+2)\left(1+x^{2}\right)-4(\beta+2) x^{2}\right. \\
\left.-4(\beta+2) \alpha x+2 \alpha+\alpha^{2}\right)
\end{gathered}
$$

The eigen function from ground state condition can be plot from the solution of $X_{0 ; l}$, the eigen function can be seen at Figure (1) bellow,

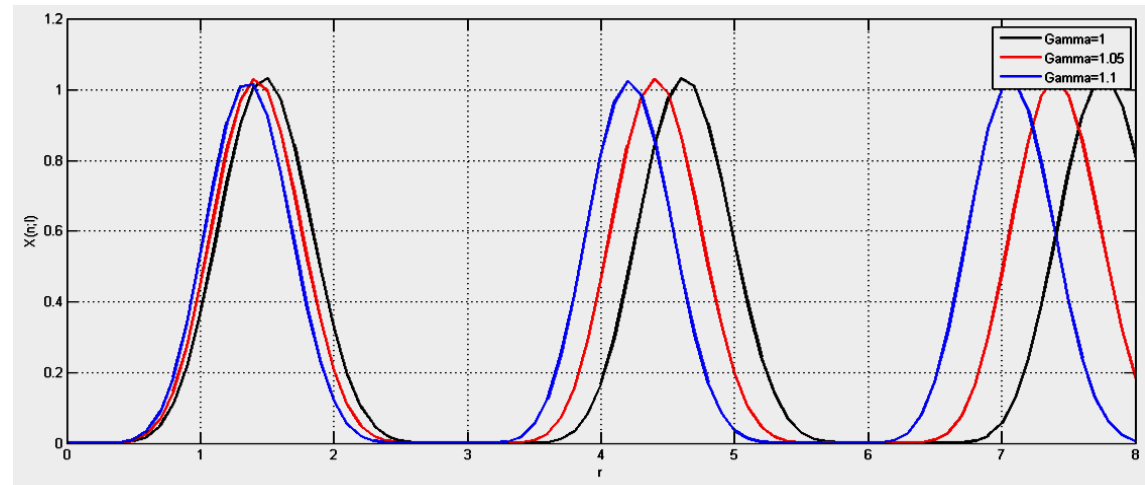

Figure 2. Eigen function with when groundstate condition

And the eigen function for first energy, can be seen at Figure (2) bellow,

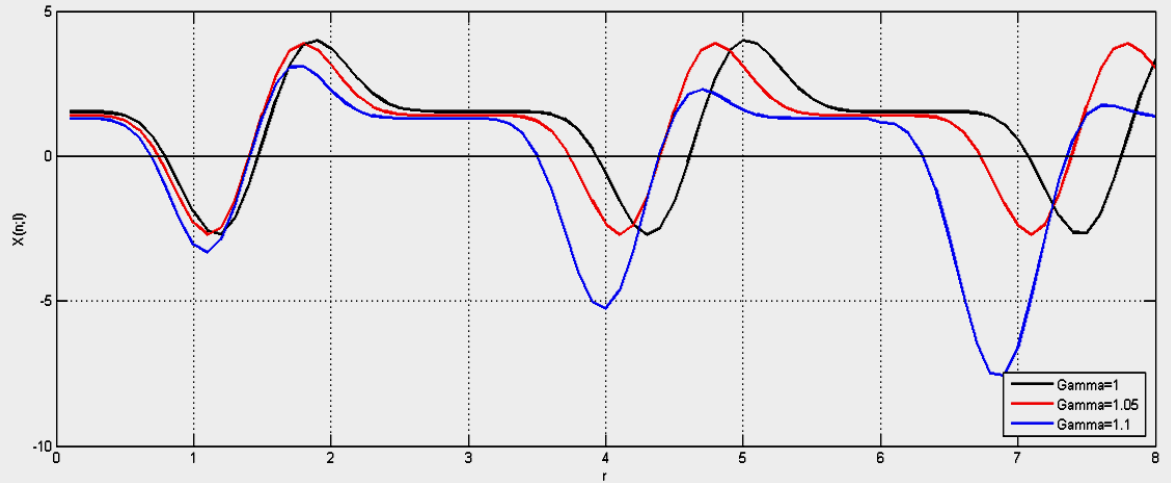

Figure 3. Eigen function with with $\gamma$ variation

Wave function explain about probability density to find of electron, because statistical interpretation about wave function basicly show that the result of measuring in the quantum system unpredictable. From the Figure (2) that explain about ground 
state condition, we can see that with the increase of gamma, not give anychange for amplitude, but just there is displacement of phase. That means electron can be find on the groundstate condition. If the amplitude increase that mean the probability to find electron of course increase. So from the graph we can say that if the value of gamma increase so the probability density also increase. Fom the graph can be know that by given a little perturbation, amplitudo increase only on the graph when the $\gamma$ value equal 1,1. But for $\gamma$ value equal 1 and 1,05 only displacement happening without increasing amplitudo.

\section{Solution of Angular Dirac Equation for Non-central Potential using Romanovsky Polynomials}

The angular dirac equation with non-central potential given as,

$-\left[\frac{\cot \theta}{P(\theta)} \frac{\partial P(\theta)}{\partial \theta}+\frac{1}{P(\theta)} \frac{\partial^{2} P(\theta)}{\partial \theta^{2}}+\frac{1}{\phi(\varphi)} \frac{1}{\sin ^{2} \theta} \frac{\partial^{2} \phi(\varphi)}{\partial \varphi^{2}}\right]+(E+M)\left(\frac{v(v+1)}{\sin ^{2} \theta}-2 q \cot \theta\right)=\lambda$

Where,

$-\frac{1}{\phi(\varphi)} \frac{\partial^{2} \phi(\varphi)}{\partial \varphi^{2}}=m^{2}$

The solution of equation (32) given as,

$\phi=\sqrt{\frac{1}{2 \pi}} e^{i m \varphi}$

By inserting equation (33) into (34) and with $\lambda=l(l+1)$ we obtain

$\frac{\partial^{2} P(\theta)}{\partial \theta^{2}}+\cot \theta \frac{\partial P(\theta)}{\partial \theta}-\left\{\frac{m^{2}}{\sin ^{2} \theta}+(E+M)\left(\frac{v(v+1)}{\sin ^{2} \theta}-2 q \cot \theta\right)-l(l+1)\right\} P(\theta)=0$

By setting $\cot \theta=x$ we have,

$\left(1+x^{2}\right) \frac{d^{2} P(\theta)}{d x^{2}}+x \frac{d P(\theta)}{d x}-\left\{m^{2}+(E+M) v(v+1)-\frac{(E+M) 2 q x}{\left(1+x^{2}\right)}-\frac{l(l+1)}{\left(1+x^{2}\right)}\right\} P(\theta)=0$

By using equation (20) the solution of equation (35) is given as,

$$
\begin{aligned}
& \left(x^{2}+1\right) \frac{\partial^{2} D_{n}^{(p, q)}(x)}{\partial x^{2}}+(2 \beta x-\alpha+x) \frac{\partial D_{n}^{(p, q)}(x)}{\partial x}-\left[\frac{\beta^{2}-\beta-\frac{\alpha x}{2}+\alpha \beta x-\frac{\alpha^{2}}{4}-(E+M) 2 q x-l(l+1)}{\left(x^{2}+1\right)}+\right. \\
& \left.m^{2}+(E+M) v(v+1)-\beta^{2}\right] D_{n}^{(p, q)}(x)
\end{aligned}
$$

By comparing equation (36) and equation (10) we obtain,

$$
\begin{aligned}
& -\frac{\alpha}{2}+\alpha \beta-(E+M) 2 q=0 \\
& \beta^{2}-\beta-\frac{\alpha^{2}}{4}-l(l+1)=0 \\
& 2(-p+1)=2(\beta+1) \text { and } q=-\alpha \\
& m^{2}+(E+M) v(v+1)-\beta^{2}=n_{l}\left(n_{l}-1\right)+2 n_{l}(1-p) \\
& \left(\beta-\frac{1}{2}\right)^{2}=\frac{2 q^{2}(E+M)^{2}}{-\left(l(l+1)+\frac{1}{4}\right) \pm \sqrt{\left(l(l+1)+\frac{1}{4}\right)^{2}+4 q^{2}(E+M)^{2}}}
\end{aligned}
$$

The value of $\beta$ and $\alpha$ obtained from equation (37a) and (37b) are given as,

$\beta=\sqrt{m^{2}+(E+M) v(v+1)}-n_{l}-\frac{1}{2}$ 
$\alpha=-\frac{(E+M) 2 q}{\sqrt{m^{2}+(E+M) v(v+1)}-n_{l}-\frac{1}{2}}$

And the orbital momentum number found from equations (37c), (37d) and (37e) together with equation (38a) given as,

$l=\sqrt{\left(\sqrt{m^{2}+(E+M) v(v+1)}+n_{l}+\frac{1}{2}\right)^{2}-\frac{2 q^{2}(E+M)^{2}}{\left(\sqrt{m^{2}+(E+M) v(v+1)}+n_{l}+\frac{1}{2}\right)^{2}}}-\frac{1}{2}$

By using equation (15) and (20) we can find angular wave function, are showed bellow,

$w(x)=\left(1+x^{2}\right)^{\sqrt{m^{2}+(E+M) v(v+1)}-n_{l}-\frac{1}{2}} e^{\frac{(E+M) 2 q}{\sqrt{m^{2}+(E+M) v(v+1)}-n_{l}-\frac{1}{2}}} \tan ^{-1}(x)$

And

$D_{n}^{(p, q)}(x)=\mathcal{R}_{n}^{(-\beta,-\alpha)}(x)=\frac{1}{\left(1+x^{2}\right)^{\beta} e^{-\alpha \tan ^{-1}(x)}} \frac{d^{n}}{d x^{n}}\left(\left(1+x^{2}\right)^{\beta} e^{-\alpha \tan ^{-1}(x)}\right)$

And the result of the wave function of the angular vave function is

$X_{n ; l}=\sqrt{\left(1+x^{2}\right)^{\beta} e^{-\alpha \tan ^{-1}(x)}} \mathcal{R}_{n}^{(-\beta,-\alpha)}(x)$

Some the solving of equation (41) and (42) are

$\mathcal{R}_{0}{ }^{\left(-\beta_{0},-\alpha_{0}\right)}=1$

$X_{n ; l}=\sqrt{\left(1+x^{2}\right)^{\beta} e^{-\alpha \tan ^{-1}(x)}}$

$\mathcal{R}_{1}{ }^{\left(-\beta_{1},-\alpha_{1}\right)}=2 x(\beta+1)-\alpha$

$X_{n ; l}=\sqrt{\left(1+x^{2}\right)^{\beta} e^{-\alpha \tan ^{-1}(x)}}(2 x(\beta+1)-\alpha)$

$\mathcal{R}_{2}{ }^{\left(-\beta_{2},-\alpha_{2}\right)}=4(\beta+2)^{2} x^{2}+2(\beta+2)\left(1+x^{2}\right)-4(\beta+2) x^{2}-4(\beta+2) \alpha x+2 \alpha+$ $\alpha^{2}$

$$
\begin{gathered}
X_{n ; l}=\sqrt{\left(1+x^{2}\right)^{\beta} e^{-\alpha \tan ^{-1}(x)}}\left(4(\beta+2)^{2} x^{2}+2(\beta+2)\left(1+x^{2}\right)-4(\beta+2) x^{2}\right. \\
\left.-4(\beta+2) \alpha x+2 \alpha+\alpha^{2}\right)
\end{gathered}
$$

From the solve special function and by using the variation ov value $v, \mathrm{q}$, and $\mathrm{E}$ we can get some angular wave function (Table. 5). The result of angular wave function can be visualizated on the form of angular wave function (Figure. 3)

Table 5. Polinomial Romanovski and the corelation with angular wave function for non

\begin{tabular}{|c|c|c|c|c|c|c|c|c|c|}
\hline No & $\mathrm{n}$ & $\mathrm{m}$ & $\tau$ & $\sigma$ & $\mathrm{E}$ & $\mathrm{M}$ & 1 & $\mathcal{R}_{1}^{\left(-\beta_{1},-\alpha_{1}\right)}(\theta)$ & $P_{l}^{m}$ \\
\hline 1. & 1 & 1 & 0 & 0 & 2 & 1 & 2 & $-3 \cot \theta$ & $-3 \cot \theta\left(1+\cot ^{2} \theta\right)^{-1.25}$ \\
\hline \multirow[b]{2}{*}{2.} & \multirow[b]{2}{*}{1} & \multirow[b]{2}{*}{1} & \multirow[b]{2}{*}{0} & \multirow[b]{2}{*}{1} & \multirow[b]{2}{*}{-2.3} & \multirow{2}{*}{1} & \multirow[b]{2}{*}{1.7} & $-3 \cot \theta$ & $(-3 \cot \theta-1.05)$ \\
\hline & & & & & & & & -1.05 & $\left.\cot ^{2} \theta\right)^{-1.25} e^{-1.05 \tan ^{-1}(\cot \theta)}$ \\
\hline \multirow{2}{*}{3.} & \multirow{2}{*}{1} & \multirow{2}{*}{1} & \multirow{2}{*}{0} & \multirow{2}{*}{2} & \multirow{2}{*}{-0.8} & \multirow{2}{*}{1} & \multirow{2}{*}{0.5} & $-3 \cot \theta$ & $(-3 \cot \theta-1.05)$ \\
\hline & & & & & & & & +0.27 & $\left.\cot ^{2} \theta\right)^{-1.25} e^{0.27 \tan ^{-1}(\cot \theta)}$ \\
\hline 4. & 1 & 1 & 1 & 0 & 5.4 & 1 & 4.7 & $-8.44 \cot \theta$ & $-8.44 \cot \theta\left(1+\cot ^{2} \theta\right)^{-2.12}$ \\
\hline 5. & 1 & 1 & 2 & 0 & -1 & 1 & 2 & $-3 \cot \theta$ & $-3 \cot \theta\left(1+\cot ^{2} \theta\right)^{-1.25}$ \\
\hline 6. & 1 & 1 & 1 & 1 & 3.4 & 1 & 4.1 & $\begin{array}{l}-9.28 \cot \theta \\
+1.91\end{array}$ & $\begin{array}{l}(-9.28 \cot \theta+1.91) \\
\left.\cot ^{2} \theta\right)^{-2.32} e^{1.91 \tan ^{-1}(\cot \theta)}\end{array}$ \\
\hline
\end{tabular}
sentral potential 


\begin{tabular}{lllllllllll}
\hline 7. & 1 & 1 & 2 & 2 & -1.1 & 1 & 3.1 & $\begin{array}{l}-\cot \theta \\
-0.43\end{array}$ & $\begin{array}{l}(-\cot \theta-0.43) \\
\left.\cot ^{2} \theta\right)^{-0.75} e^{-0.43 \tan ^{-1}(\cot \theta)}\end{array}$
\end{tabular}$\quad(1+$

The angular wave function can be showed by the picture bellow,

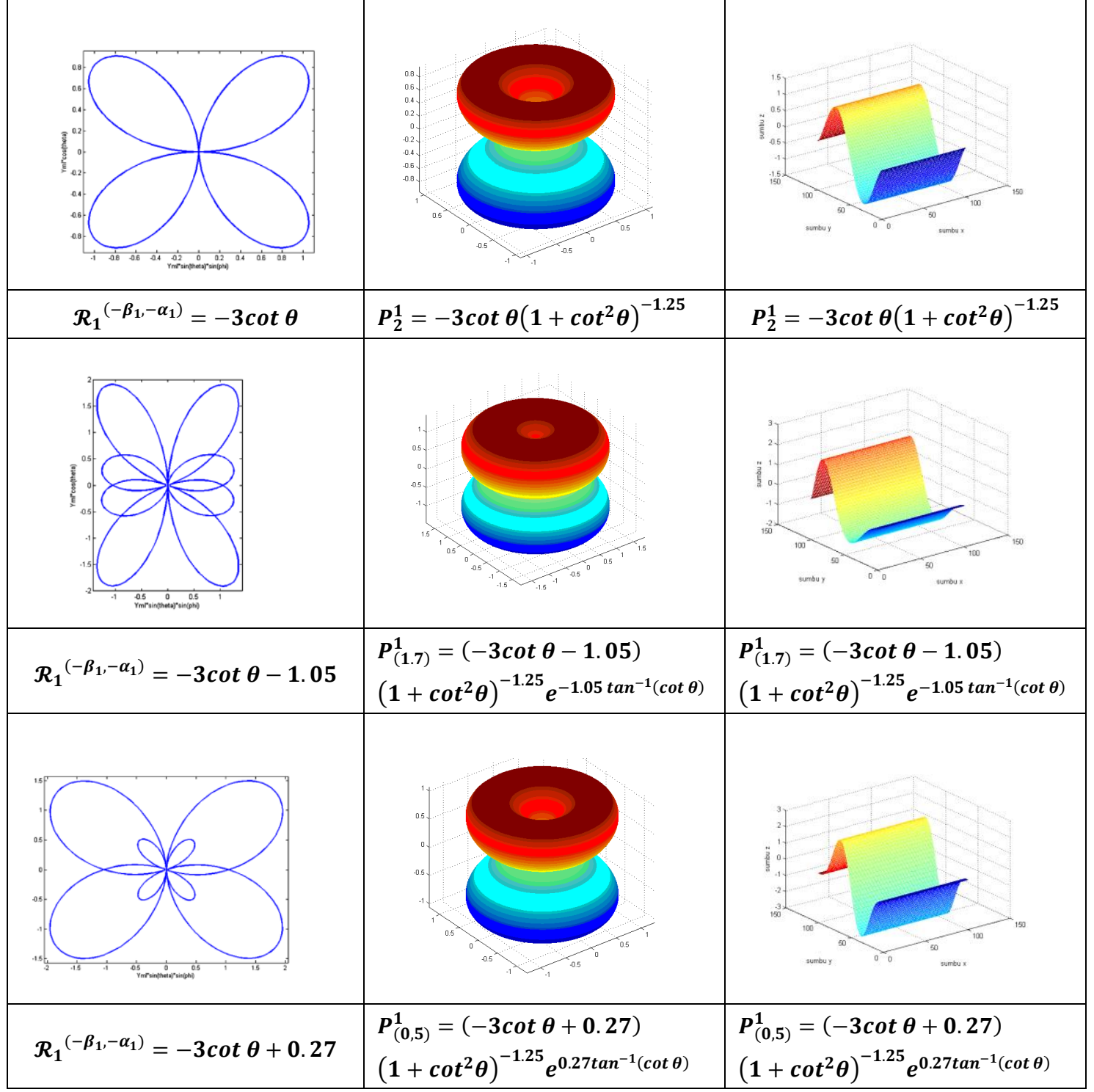

Figure 3. Visualisation of angular wave function on 3 and 2 dimension with the variation of $\mu$

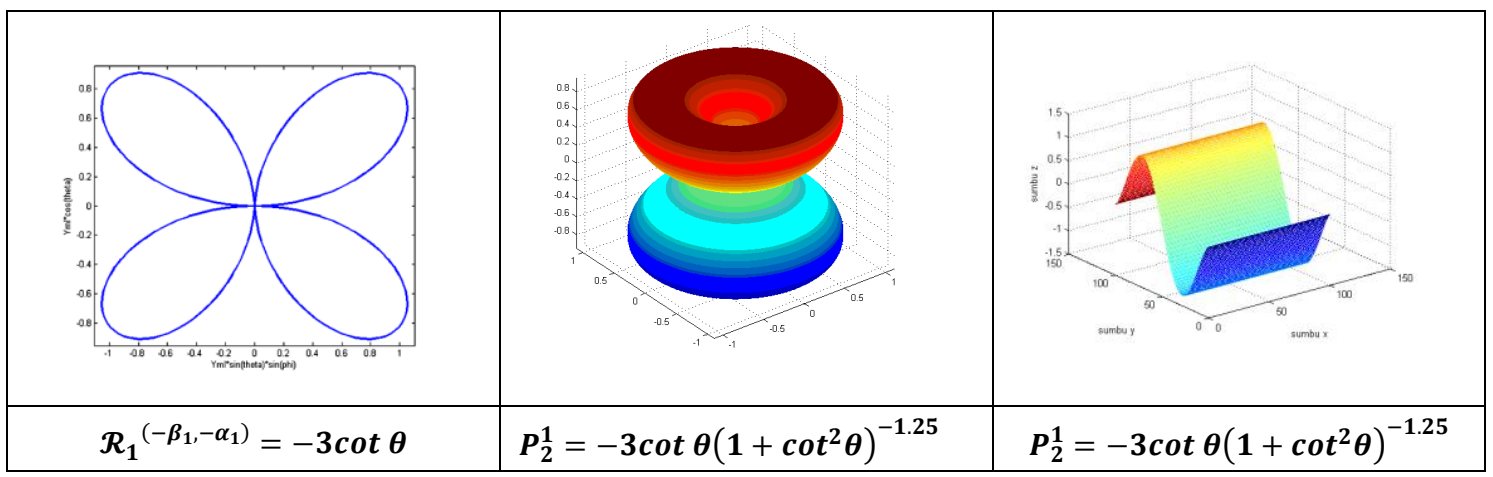




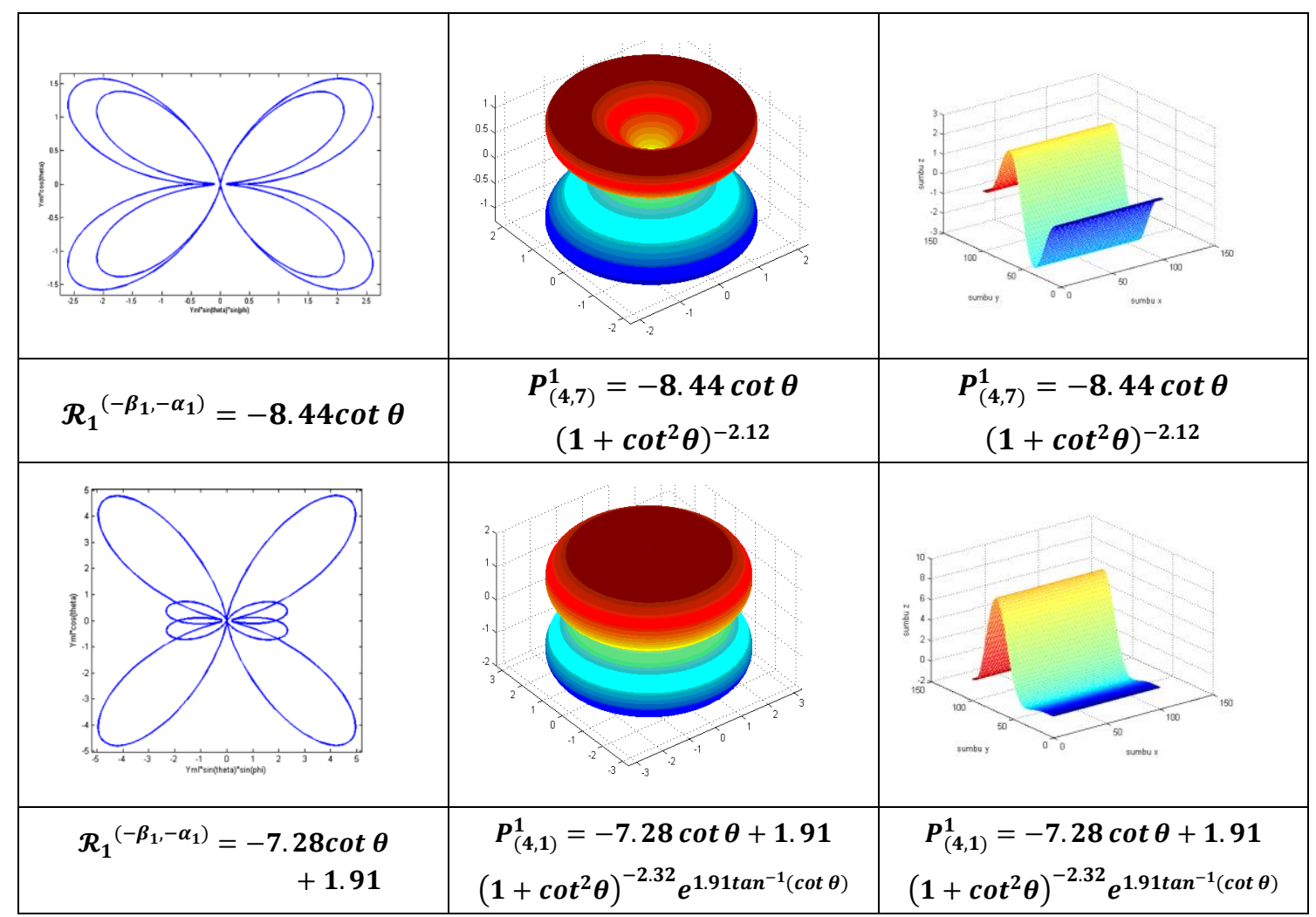

Figure 4. Visualisation of angular wave function on 3 and 2 dimension with the variation of $\sigma$

The form of angular wave function related with direction of angular momentum and discribing deppending of probability density to the angular. General definition of angular wave function same with radial wave function, but both have differences. Radial wave function about far or near electron from nucleus, if angular wave function corellation with around of electron.

For the value of $m_{l}=0$, electron can be found on the $\mathrm{z}$ axis. But for the value $m_{l}=$ \pm 1 , electron can be found on the $\mathrm{x}$ and $\mathrm{y}$ shape. If $m_{l}=1$, the electron counter clock wise, but if $m_{l}=-1$ the electron opposite.

The form of angular wave function it influenced by the value of $v$ and $\mathrm{q}$ that give different influence to form of angular wave function. Increasing q value but the $v$ is constan, the noise that be given by Rosen Morse potential can make smaller wave function on the $\mathrm{x}, \mathrm{y}$, and $\mathrm{z}$ direction. But if the increasing $v$ value with the $\mathrm{q}$ constan, the noise that be given by Rosen Morse potential can make biger wave function. If $v$ and $\mathrm{q}$ value not constan, all the wave funtion can be smaller.The changes of angular wave function to the $v$ and q value explained that the noise from Rosen Morse potential make change to probability on the angular function, but not influence to the direction of electron circle.

\section{Conclusion}

In this paper be explained the numerical solution of Dirac equation for Rosen Morse potential plus Rosen Morse potential with the centrifugal term in term of Romanovski 
polynomials. The energy spectrum is obtained in the closed form and the wave function obtained is expressed in terms of Romanovski polynomials. With a little increasing of of $\gamma$ can make large change of energy eigen value.

\section{References}

Azizi, M., Salehi, N., \& Rajabi, A. A. (2013). Exact Solution of the Dirac Equation for the Yukawa Potential with Scalar and Vector Potentials and Tensor Interaction. ISRN High Energy Physics, 2013, 1-6. doi:10.1155/2013/310392

Bakkeshizadeh, S., \& Vahidi, V. (2012). Exact Solution of the Dirac Equation for the Coulomb Potential Plus NAD Potential by Using the Nikiforov-Uvarov Method. Adv. Studies Theor. Phys, 6(15), 733-742.

Cari \& Suparmi (2012) Approximate Solution of Schrodinger Equation for Hulthen Potential plus Eckart Potential with Centrifugal Term in terms of Finite Romanovski Polynomials vol 2, International Journal of Applied Physics and Mathematics, p 159-161.

Cari, Suparmi and H.Marini (2012) Penentuan Spektrum Energi dan Fungsi Gelombang Potensial Morse dengan Koreksi Sentrifugal Menggunakan Metode SWKB dan Operator SUSY vol 2 Indonesian Journal of Applied Physics, p 112.

Castillo, David. (2009). Exactly Solvable Potentials and Romanovski Polynomials in Quantum Mechanics. Instituto de F'1sica Universidad Aut'onoma de San Luis Potos. arXiv:0808.1642v2 [math-ph].

Eshghi, M., Hamzavi, M., \& Ikhdair, S. M. (2012). Exact solutions of a spatially dependent mass Dirac equation for Coulomb field plus tensor interaction via laplace transformation method. Advances in High Energy Physics, 2012.

Reity, Rubish and Myhalyna. (2014). The WKB Method for the Dirac Equation with Vector-Scalar Potentials in $2+1$ and $3+1$ Dimensions vol. 50, Proceedings of Institute of Mathematics of NAS of Ukraine, p 1429-1434.

Ikot, A. N., \& Akpabio, L. E. (2010). Approximate solution of the Schrodinger equation with Rosen-Morse potential including the centrifugal term. Applied Physics Research, 2(2), 202-208.

Sadeghi, Pahlavani, Naderi and Banijamali. (2005). Solution of the Relativistic Dirac Equation for Woods-Saxon potential Proceedings QPF Sept. 22.

Villalba, V. M. (2005). Exact solution of the Dirac equation in the presence of a gravitational instanton. Journal of Physics: Conference Series, 24, 136-140. doi:10.1088/1742-6596/24/1/016

Weber, H. J. (2007). Connections between Romanovski and other polynomials. Central European Journal of Mathematics, 5(3), 581-595. doi:10.2478/s11533-0070014-4 